\title{
Critical phenomena in disc-percolation model and its application to relativistic heavy ion collisions
}

\author{
Ke Hongwei, ${ }^{1}$ Xu Mingmei, ${ }^{1,2}$ and Liu Lianshou ${ }^{1,2, \text { 田 }}$ \\ ${ }^{1}$ Institute of Particle Physics, Huazhong Normal University, Wuhan 430079, China \\ ${ }^{2}$ Key Lab of Quark and Lepton Physics (Huazhong Normal University), Ministry of Education, China
}

\begin{abstract}
Through studying the critical phenomena in continuum-percolation of discs, we find a new approach to locate the critical point, i.e. using the inflection point of $P_{\infty}$ as an evaluation of the percolation threshold. The susceptibility, defined as the derivative of $P_{\infty}$, possess finite-size scaling property, where the scaling exponent is the reciprocal of $\nu$ - the critical exponent of correlation length. The possible application of this approach to the study of the critical phenomena in relativistic heavy ion collisions is discussed. The critical point for deconfinement can be extracted by the inflection point of $P_{\mathrm{QGP}}$ - the probability for the event with QGP formation. The finite-size scaling of its derivative can give the critical exponent $\nu$, which is a rare case that can provide an experimental measure of a critical exponent in heavy ion collisions.
\end{abstract}

PACS numbers: 25.75.Nq, 24.10.Lx, 64.60.ah

\section{INTRODUCTION}

The general feature of the phase diagram of strongly interacting matter has become increasingly well established [1]. Following a region of crossover around a temperature of 170 - $190 \mathrm{MeV}$ at zero baryon chemical potential $\mu_{B}$, increasing $\mu_{B}$ leads to a critical point, beyond which the system shows a first order transition from confined to deconfined phase.

Recently, the interest in QCD critical point progressively rises. With the plan of RHIC low energy scan and GSI new facility, the study of relativistic heavy ion collisions becomes concentrating on searching for the critical point and observing the relevant critical phenomena, in particular measuring the critical exponents.

On the theoretical side, studying the critical phenomena in chiral symmetry restoration is available [2]. Many discussions about universality have been active [3]. The chiral condensate and Polyakov loop are proposed as order parameters for chiral restoration and color deconfinement, respectively. However, it is a pity that both of these variables can not be directly measured by experiment. What observable should we measure, how to locate the critical point and extract the corresponding critical exponents in heavy ion collisions have not been clear so far.

On the experimental side, what we observed in heavy ion collisions is the deconfined partonic degree of freedom [4]. Despite the fact that confinement is a long standing problem not solved by theory, studying the critical phenomena in deconfinement phase transition is more realistic for experiments. Since chiral symmetry is hard to measure and deconfinement is easier to observe, we propose to phenomenologically study the critical phenomena from the deconfinement aspect and give some

*Electronic address: liuls@iopp.ccnu.edu.cn hints for experiments.

Principally speaking, it is impossible to get the critical point because of the limited system size in relativistic heavy ion collisions. In this paper, by means of studying the critical phenomena in finite-size continuumpercolation of disks, we find that $P_{\infty}$, the probability for event with infinite cluster, has an inflection point, which is a good approximation for the critical point. Finitesize scaling method is further used to extract the critical exponents $\nu$ from the distribution of susceptibility. Since percolation has some resemblance with deconfinement [5], a possible application of the method used in percolation to the study of the critical phenomena in relativistic heavy ion collisions is discussed.

\section{THE CONTINUUM-PERCOLATION OF DISC}

The continuum-percolation problem has various formulations, among which the problem of spheres is most popular. Equally sized spheres are placed at random in a substrate. The spheres support the transport and the substrate does not. When we put enough spheres in the substrate, the overlapping spheres form an infinite cluster, and the system is able to support a longrange current. This model has been well studied and was used to describe hopping conduction in doped semiconductors [6] and phase transitions in ferromagnetics [7]. Such a model together with its critical behavior also bare some resemblance with the transition from hadron gas to quark-gluon plasma.

In QCD, hadrons are color-singlet bound states of more basic colored objects — quarks and gluons. Hadronic matter, consisting of colorless constituents of hadronic dimensions, can turn at high temperature and/or density to a quark-gluon plasma of colored quarks and gluons as constituents in a much larger volume. This deconfinement transition leads to a color-conducting state and thus is the QCD counterpart of the insulator-conductor transi- 
tion in atomic matter. Suppose hadrons have an intrinsic size. At low density, we have a hadron gas. When this becomes so dense that the formation of an infinite cluster occurs, it turns to a quark-gluon plasma. In this case, the connectivity (cluster formation) determines the different states of many-body systems. The lesson learned from spin systems also indicates that cluster formation and associated critical behavior are the more general feature [5].

\section{A. Model parameters}

In relativistic heavy ion collisions the incoming nuclei become discs of vanishing thickness due to Lorentz contraction and the collision region is two-dimensional. So we turn to study two-dimensional percolation.

In a two-dimensional system, spheres become discs. Discs with radius $a$, called cells, distribute randomly in the system of a big disc with radius $R$. The ratio $R / a$ determines the system size and is denoted by $s$. The control variable for percolation is $\eta$ - the ratio of the total area of all the cells and the area of the big disc, i.e.

$$
\eta=\frac{N \pi a^{2}}{\pi R^{2}}=\frac{N a^{2}}{R^{2}}
$$

where $N$ is the number of cells. The increase of cell number $N$ leads to the increase of $\eta$. The value of $\eta$ at which an infinite cluster appears is the critical point and is denoted by $\eta_{c}$.

The definition for infinite cluster varies. In finite system a cluster spanning the system is called an infinite cluster. Let $\left(r_{i}, \theta_{i}\right)$ be the polar coordinates of cell $i$. If $r_{i} \in[R-a, R]$ we call the cell $i$ as a boundary cell. If cells $i$ and $j$ are two boundary cells belonging to the same cluster, and $\left|\theta_{i}-\theta_{j}\right| \geq \theta_{0}$ then we say the current cluster is infinite. Here $\theta_{0}$ is a parameter.

Thus the control variable is $\eta$ and the model parameters are $s$ and $\theta_{0}$.

\section{B. Locating the critical point}

When $s$ and $\theta_{0}$ take certain values respectively, the probability for the event with infinite cluster, denoted by $P_{\infty}$, is an increasing function of $\eta$. For infinite system size, $P_{\infty}=0$ holds for all $\eta<\eta_{c}$. When $\eta \geq \eta_{c}, P_{\infty}$ picks up and sharply increases to unity. System of infinite size is unrealistic. $P_{\infty}$ for finite system of three different sizes are measured and shown in Fig. 1.

Different parameters, e.g. $\theta_{0}=90^{\circ}, 135^{\circ}$ and $175^{\circ}$, are tried and the induced systematic errors are shown as error-bars in the figures. The solid curves in Fig. 1 are the fitting result by using the function

$$
P_{\infty}(\eta)=\frac{1+\tanh \left[c_{1}\left(\eta-c_{2}\right)\right]}{2} .
$$

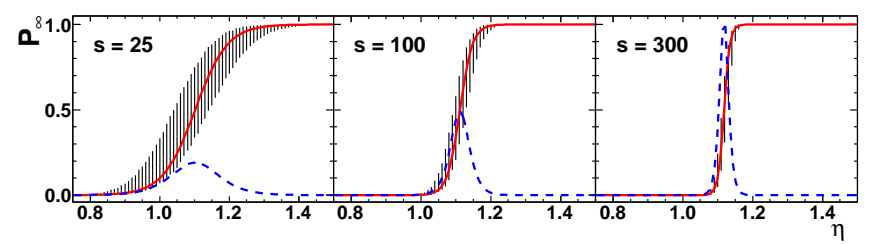

FIG. 1: (Color online) $P_{\infty}$ for different system size. From left to right the system sizes are $25,100,300$, respectively. The error bar shown is systematic error induced by the variation of parameter $\theta_{0}$. Statistical errors are small and not shown. The dashed curves are the susceptibility defined in Eq. (4)

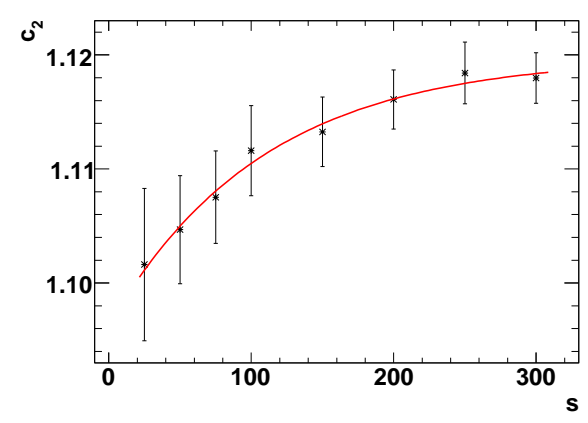

FIG. 2: (Color online) The behavior of $c_{2}$ versus system size. A tendency to saturation can be seen.

It can be seen that for all three cases the fitting function always lies within the systematic errors. The fitting function, i.e. Eq.(2), has an inflection point at $\eta=c_{2}$. As $s$ increases, $P_{\infty}$ tends to a step function and the inflection point $c_{2}$ tends to $\eta_{c}$. For finite system size, the inflection point $\eta=c_{2}$ can be used as an evaluation of $\eta_{c}$.

The behavior of $c_{2}$ versus different system size is shown in Fig. 2. A tendency to saturation can be seen. The saturation value of $c_{2}$ is $1.1198 \pm 0.0047$, i.e. the percolation threshold is $\eta_{c}=1.1198 \pm 0.0047$.

For a nucleus-nucleus collision, the contraction induced by relativistic motion makes the colliding nucleus like a disc, in which disc-like nucleons percolate. For central $\mathrm{Au}-\mathrm{Au}$ collision, the radius of the interacting region is about $7 \mathrm{fm}$ and the hard core of each nucleon is about $0.1 \mathrm{fm}$, resulting in a system size of about 70 . The inflection point for $s=70$ is about 1.1054. Using it as an approximation for $\eta_{c}$, the error is $1.3 \%$. So using the inflection point as an approximation for critical point is easy to measure and exact enough.

In heavy ion collisions, when hadrons connect to form infinite cluster, color conducts in the system and quark gluon plasma is formed. If the formation of QGP can be signaled in each collision, then the probability for the event with infinite cluster, denoted by $P_{\infty}$, becomes the probability for the collision with QGP $-P_{\mathrm{QGP}}$. $P_{\mathrm{QGP}}$ should be measurable in experiment, provided that an available signal is found, and thus in principle the critical 


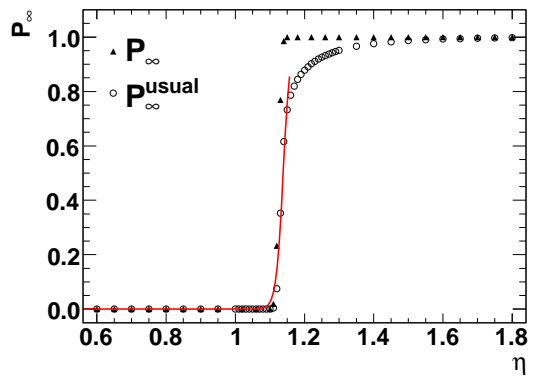

FIG. 3: (Color online) $P_{\infty}^{\text {usual }}$ (open circles) as a function of $\eta$ with system size $s=1000$. The solid curve is the fit of the lower part to Eq. (3). For comparison the $P_{\infty}$ for $s=1000$ is also shown as triangles.

point can be obtained according to the inflection point of $P_{\mathrm{QGP}}$.

$P_{\infty}$ is also referred to by some authors $[8]$ as percolation cumulant, in analogous to the Binder cumulant [9], which will intersect at the critical point for different system sizes. Extracting the intersection point of $P_{\infty}$ is a method to estimate the critical point. However, to use the inflection-point method proposed above only needs the collisions of one kind of ion at various energies, while to use the intersecting-point method need the collision at different energies of more than one kind of ion. Therefore, the inflection-point method is more realistic and the value of $\eta_{\mathrm{c}}$ obtained for system sizes as small as $\mathrm{Pb}$ $(s=71), \mathrm{Au}(s=70)$ is a good estimation with error about $1.3 \%$.

The inflection-point method is also applicable for the P-infinity defined in another way. In Ref. [10] P-infinity is defined as the probability of a cell belonging to an infinite cluster, which will be denoted by $P_{\infty}^{\text {usual }}$ in the following. Fig. 3 shows $P_{\infty}^{\text {usual }}$ as a function of $\eta$ when $s=1000$. In case of $s=1000$, the system is a big one and $P_{\infty}$ rises like a step function, cf. Fig. 3. but $P_{\infty}^{\text {usual }}$ has a step function sharp rise only at the bottom side $\left(P_{\infty}^{\text {usual }}=0\right)$, while at the upper side $P_{\infty}^{\text {usual }}$ tends to unity smoothly.

In this case, we could only fit the left part of $P_{\infty}^{\text {usual }}(\eta)$ to Eq. (2), or alternatively, to a modified fitting function

$$
P_{\infty}^{\text {usual }}(\eta)=c_{1}\left[c_{2}+\tanh \left(c_{3}\left(\eta-c_{4}\right)\right)\right] .
$$

The fitting is shown as solid curve in Fig. 3 The inflection point of this half-fitted curve can also be used as an approximation of the critical point.

\section{Susceptibility and the critical exponent $\nu$}

Susceptibility is defined by the response of the system to small external forces. We define the susceptibility in percolation as the derivative of $P_{\infty}$ with respect to the control variable $\eta$, i.e.

$$
\kappa(\eta, s)=\frac{\partial P_{\infty}(\eta, s)}{\partial \eta}
$$

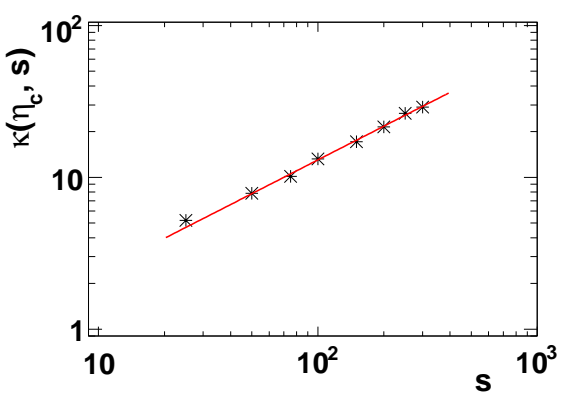

FIG. 4: (Color online) The distribution of susceptibility $\kappa$ at critical point $\eta_{c}$ as a function of system size $s$. The solid line is a fitting by Eq. (8).

$\kappa$ shows a peak at the inflection point of $P_{\infty}$, see the dashed line in Fig. 1. Since $P_{\infty}$ becomes a step function when $s$ tends to infinity, the peak of $\kappa$ will become higher and narrower and finally diverge at $\eta_{c}$.

In realistic case, the system is of finite size determined by the size of the colliding nuclei. The finite-size scaling method 11, investigating the scaling of quantities at $\eta_{c}$ as a function of system size, is adopted to extract values for the critical exponents.

The finite-size scaling of $P_{\infty}$ suggests $[12$ ]

$$
P_{\infty}=\Phi\left[\left(\eta-\eta_{c}\right) s^{1 / \nu}\right] \quad \text { for large } s, \quad \eta \rightarrow \eta_{c} .
$$

Denoting $X=\left(\eta-\eta_{c}\right) s^{1 / \nu}, \Phi(X)$ is some function of $X$. $\nu$ is the critical exponent of correlation length $\xi$, i.e.

$$
\xi \propto\left|\eta-\eta_{c}\right|^{-\nu} \quad \text { for } \eta \rightarrow \eta_{c} .
$$

Then, the susceptibility of $P_{\infty}$ is

$$
\kappa(\eta, s)=\frac{\partial P_{\infty}(\eta, s)}{\partial \eta}=s^{1 / \nu} \frac{\mathrm{d} \Phi}{\mathrm{d} X} .
$$

When $\eta=\eta_{c},\left.\frac{\mathrm{d} \Phi}{\mathrm{d} X}\right|_{X=0}$ is a constant and

$$
\kappa\left(\eta_{c}, s\right) \propto s^{1 / \nu} \quad \text { for large } s .
$$

So the divergence behavior of $\kappa$ near $\eta_{c}$ is related with the critical exponent of correlation length.

Using the critical point $\eta_{c}$ extracted from $P_{\infty}$, we evaluate $\kappa\left(\eta_{c}, s\right)$ as a function of $s$, shown in Fig. 4. Fitting it to Eq. (8) we obtain $1 / \nu=0.739 \pm 0.041$, $\nu=1.353 \pm 0.075$. The exponent $\nu$ obtained is within $1.5 \%$ difference with the result from other calculations for two-dimension percolation [10].

The critical exponent of correlation length plays a special role in the theory of critical phenomena, because the scaling behavior of other quantities depends on the relative magnitude of correlation length and system size. Hence, the critical exponent of correlation length appears in the scaling relation of various quantities. By definition, correlation length is the distance at which correlation function reduces to $1 / \mathrm{e}$ [10]. However, correlation function usually has non-monotonic behavior, e.g. in 


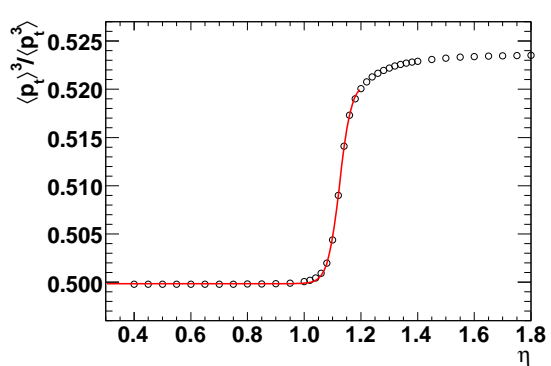

FIG. 5: (Color online) $D_{3}$ as a function of $\eta$ with system size $s=500$. Red line is a fitting by Eq.(3).

case of liquid, correlation function shows damping oscillation [13], which makes the correlation length hard to be measured. Now we see that using the scaling behavior of susceptibility the critical exponent of correlation length can easily be obtained [12].

\section{APPLICATION TO RELATIVISTIC HEAVY ION COLLISION}

Recently, a kind of scaled third order moment of transverse momentum [14]

$$
D_{3}=\frac{\left\langle p_{t}\right\rangle^{3}}{\left\langle p_{t}^{3}\right\rangle}
$$

has been proposed as a possible signal of critical-point (CP) of QCD phase diagram. By imposing a temperature gradient to the two dimensional continuum percolation of discs and assuming the transverse momentum for each cell takes the thermal momentum determined by the temperature, $D_{3}(\eta)$ behaves like a step function, similar to that of $P_{\infty}^{\text {usual }}(\eta)$ described above, cf. Fig. 5 and Fig. 3. This similarity makes the methods developed in Sec II applicable to $D_{3}$, i.e. we can locate the CP and determine the critical exponent of a susceptibility related to $D_{3}$.

A typical result for $D_{3}$ with a half-fit to Eq. (3) is shown in Fig. 5 The inflection point $c_{4}$ is regarded as the approximation of critical point $\eta_{c}$. With the increasing of system size, the inflection point $c_{4}$ has an asymptotic behavior which is shown in Fig. 6. The saturation value indicates $\eta_{c}=1.124$.

Similar to Eq. (44) we define the susceptibility related to $D_{3}$ as

$$
\kappa^{\prime}(\eta, s)=\frac{\partial D_{3}(\eta, s)}{\partial \eta}
$$

and a critical exponent $\nu^{\prime}$ for this thermal system as

$$
\kappa^{\prime}\left(\eta_{c}, s\right) \propto s^{1 / \nu^{\prime}} .
$$

Investigating the finite-size scaling of $\kappa^{\prime}, 1 / \nu^{\prime}=0.642$ is obtained, c.f. Fig. 7 .

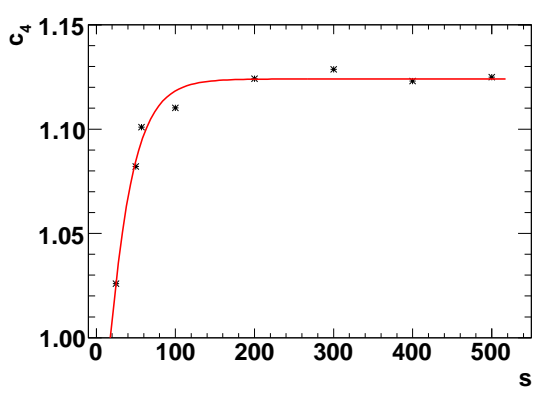

FIG. 6: (Color online) The behavior of $c_{4}$ versus different system size. A saturation is seen.

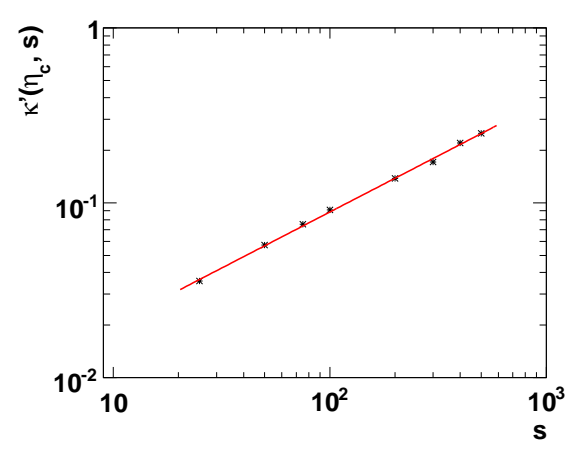

FIG. 7: (Color online) The distribution of susceptibility $\kappa^{\prime}$ at critical point $\eta_{c}$ as a function of system size $s$. The solid line is a fitting to Eq. (11).

\section{CONCLUSION}

We study the critical phenomena in continuum percolation of discs. It is shown that using the inflection point of $P_{\infty}$ or $P_{\infty}^{\text {usual }}$ as an estimation for percolation threshold is a good approximation. The critical exponent $\nu$ is extracted from the distribution of susceptibility by using finite-size scaling method.

For heavy ion collisions, different colliding nuclei have been tried, e.g. $\mathrm{Pb}, \mathrm{Au}, \mathrm{Cu}, \mathrm{S}, \mathrm{C}$ etc., which play the role of system size in percolation. The colliding energy plays the role of control variable. $P_{\infty}$ in percolation corresponds to the probability of events with QGP in heavy ion collision experiment, denoted as $P_{\mathrm{QGP}}$. Studying the inflection point of $P_{\mathrm{QGP}}$ as a function of $\sqrt{s_{N N}}$, the critical point can be extracted. A susceptibility defined as the derivative of $P_{\mathrm{QGP}}$ determines the critical exponent $\nu$ which is a rare case that can experimentally measure the critical exponent in heavy ion collisions.

The analogue between percolation and deconfinement is reasonable. The approach studied here is worth trying in the future study of the critical phenomena in real relativistic heavy ion collisions.

Acknowledgement This work is supported by NSFC under projects No.10775056, 10835005 and 10847131. 
[1] For a recent survey, see e.g., F. Karsch, J. Phys. Conf. Ser. 46, 122 (2006).

[2] K. Fukushima, talk given in the seminar of "The QCD Critical Point", Washington, July 28 - August 22, 2008.

[3] R. Pisarski and F. Wilczek, Phys. Rev. D 29, 338 (1984); K. Rajagopal, Quark-Gluon Plasma 2, edited by R. Hwa (World Scientific, Singapore, 1995), p. 484; J. Berges and K. Rajagopal, hep-ph/9804233 M.A. Halasz et al., hep-ph/9804290, For a review, see I. Lawrie and S. Sarbach, in Phase Transitions and Critical Phenomena, edited by C. Domb and J. Lebowitz (Academic Press, New York, 1984), Vol. 9, p. 1.

[4] J. Adams et al. (STAR Collaboration), Nucl. Phys. A757, 102 (2005); K. Adcox et al. (PHENIX Collaboration), Nucl. Phys. A757, 184 (2005).

[5] H. Satz, Nucl. Phys. A642, c130 (1998); H. Satz, Int. J. Mod. Phys. A 21, 672 (2006).

[6] B.I. Shklovskii and A.L. Efros, Electronic Properties of Doped Semiconductors, Springer, New York, 1984.

[7] A.A. Abrikosov, Spin glasses with short range interac- tion, Adv. Phys. 29, 869 (1980).

[8] S. Fortunato and H. Satz, Phys. Lett. B509, 189 (2001); Nucl. Phys. B598 601 (2001).

[9] K. Binder, D.W. Heermann, Monte Carlo Simulations in Statistical Physics, Springer-Verlag, 1988, p.40.

[10] K. Christensen and N. R. Moloney, Complexity and Criticality, Imperical College Press, London, 2005.

[11] M. Barber, Finite size scaling, in C. Domb and J. Lebowitz, editors (1993), Phase Transitions and Critical Phenomena, volume 8, Academic Press, London.

[12] D. Stauffer, A. Aharony, Introduction to percolation theory, Taylor \& Francis, 2003.

[13] N.H. March and M.P. Tosi, Introduction to liquid state physics, World Scientific Publishing Co., Singapore, 2002.

[14] Du Jiaxin, Ke Hongwei, Xu Mingmei and Liu Lianshou, The characterizing variable for critical point in momentum space, arXiv:0810.1989 nucl-th]. 